\title{
Tuberculous aneurysm of the descending thoracic aorta
}

\author{
Carlo M. Hatem, MD, a George A. Kantis, MD, ${ }^{a}$ Dimitri Christoforou, MD, Jeffrey P. Gold, MD, ${ }^{b}$ and
} Konstadinos A. Plestis, MD, Bronx, NY

$\mathrm{T}$ uberculous aortic aneurysm (TBAA), also called mycotic aneurysm, is an exceedingly uncommon condition. It occurs in the setting of disseminated tuberculosis and has a high fatality rate. We report on a case of fever of unknown origin in which TBAA ultimately developed and was successfully repaired surgically.

\section{Clinical Summary}

A 55-year-old woman with diabetes, who required hemodialysis, was admitted with fever of unknown origin. She had been having chills, night sweats, productive cough, and weight loss of 2 months' duration. An empiric treatment course with vancomycin at another hospital had failed. Her mother had recently been treated for tuberculosis. Results of the physical examination was notable for icterus, fever, tachycardia, and hypertension. She had hepatosplenomegaly and bilateral axillary and inguinal lymphadenopathy. Initial laboratory studies revealed elevated transaminase levels. The chest radiograph was clear. Sputum staining and culture for acid-fast bacilli was negative. Computed tomographic (CT) scan of the chest and abdomen revealed calcification of the aorta, para-aortic lymphadenopathy, prominent heterogeneous liver, and splenomegaly (Figure 1, A). Percutaneous liver biopsy and bone marrow biopsy revealed widespread granulomas with caseous necrosis consistent with tuberculosis. Directly observed therapy consisting of rifampin, isoniazid, pyrazinamide, and ethambutol was started with quick resolution of symptoms.

Six months later, she came to the emergency department with sharp back pain and shortness of breath. A chest radiograph revealed a retrocardiac density. CT scan of the chest without contrast medium revealed high attenuation material consistent with acute hemorrhage associated with an aneurysm involving the descending thoracic aorta and measuring $6.5 \times 5.0 \times 8.0 \mathrm{~cm}$ (Figure 1, B). At thoracotomy, a ruptured saccular aneurysm of the

From the Departments of Medicine ${ }^{\mathrm{a}}$ and Cardiothoracic Surgery, ${ }^{\mathrm{b}}$ Montefiore Medical Center, The University Hospital of the Albert Einstein College of Medicine, Bronx, NY.

Received for publication Aug 28, 2001; accepted for publication Sept 27, 2001.

Address for reprints: K. A. Plestis, MD, Montefiore Medical Park, 1575 Blondell Ave, Suite 125, Bronx, NY 10461 (E-mail: dplestis@montefiore.org).

J Thorac Cardiovasc Surg 2002;123:373-4

Copyright $(9) 2002$ by The American Association for Thoracic Surgery

$0022-5223 / 2002 \$ 35.00+0 \quad \mathbf{1 2 / 5 4 / 1 2 0 7 2 1}$

doi:10.1067/mtc.2002.120721
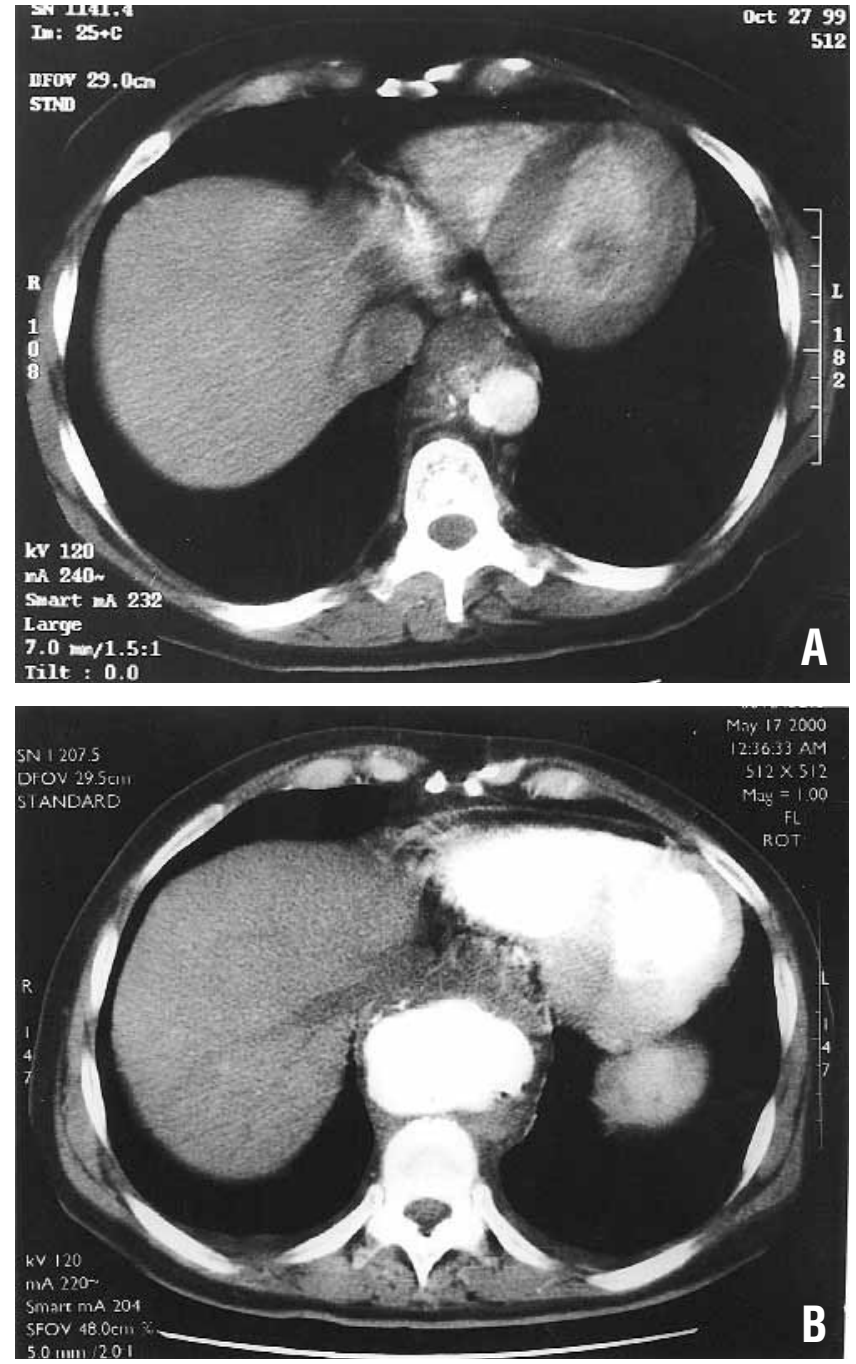

Figure 1. A, CT scan showing the descending thoracic aorta at initial presentation. B, Six months later, follow-up CT scan at the same level demonstrating a large aneurysm arising from the aorta.

distal descending thoracic aorta was identified. It was surrounded by an intense inflammatory reaction, and purulent material was noted in the aneurysmal wall. With the patient on bypass, the affected area was debrided and irrigated with antiseptic solution. The aorta was then repaired with a Dacron graft and wrapped with a piece of omentum. Pathologic examination revealed giant cells 
and tuberculous bacteria in the surgical specimen. Sensitivity testing showed no evidence of resistance. The postoperative course was uneventful and the same antituberculous regimen was continued. At follow-up 6 months later the patient was asymptomatic.

\section{Discussion}

Tuberculous aortic aneurysm is exceedingly uncommon; only 44 cases have been reported since $1945,{ }^{1,2} 19$ affecting the thoracic aorta. Involvement of the aorta with Mycobacterium bacilli can occur as a result of direct extension from an adjacent focus or via hematogenous spread. The former is believed to be the more common mechanism. However, the sequence of events is frequently difficult to determine because TBAA occurs in the setting of disseminated tuberculosis. Infection of a preexisting aneurysm with mycobacteria has not previously been reported. Atheromatous plaques have been associated with foci of tuberculous aortitis and believed to be predisposing lesions for TBAA. Patterns of clinical presentation include symptoms related to the infection such as fever, cough, night sweats, and weight loss with or without signs and symptoms related to the aneurysm itself, such as back pain, paraaortic mass, and hypovolemic shock.

Historically, the mortality rate has been high, but recent trends are toward better survival. Before 1980, 61\% died (19/31), whereas only $14 \%$ died thereafter (2/14, including the current case). Most deaths, about $70 \%$, resulted from fatal exsanguination. The improvement in survival is probably the result of several factors including more effective antituberculous regimens, closer patient follow-up with directly observed therapy programs, more frequent use of chest films and CT scans in patient care, and improvement in surgical techniques. Correlation with trends in the incidence of tuberculosis and human immunodeficiency virus cannot be established because of the paucity of numbers. Only 2 cases of TBAA have been reported in patients with human immunodeficiency virus. ${ }^{3}$

Medical treatment alone is not sufficient. In several cases, including this one, the aneurysm developed despite appropriate antituberculous therapy. ${ }^{4}$ This is probably due to poor drug penetration into necrotic tissues. Once TBAA is suspected, surgery should not be delayed because rupture could be imminent. The appropriate surgical approach varies with the type of aneurysm and the extent of damage to the vessel wall. Extra-anatomic bypass has fallen out of favor because simpler procedures can be performed. Antituberculous therapy should be initiated if the diagnosis is made intraoperatively.

Physicians should be aware of this potentially fatal condition, especially at a time when the incidence of tuberculosis is rising. Early detection is the key to successful management.

\section{References}

1. Long R, Guzman R, Greenberg H, Safneck J, Hershfield E. Tuberculous mycotic aneurysm of the aorta: review of published medical and surgical experience. Chest. 1999;115:522-31.

2. Strnad BT, McGraw JK, Heatwole EV, Clark P. Tuberculous aneurysm of the aorta presenting with uncontrolled hypertension. $J$ Vasc Interv Radiol. 2001;12:521-3.

3. Gouny P, Valverde A, Vincent D, Fadel E, Lenot B, Tricot JF, et al. Human immunodeficiency virus and infected aneurysm of the abdominal aorta: report of three cases. Ann Vasc Surg. 1992;6:239-43.

4. Ikezawa T, Iwatsuka Y, Naiki K, Asano M, Ikeda S, Kimura A. Tuberculous pseudoaneurysm of the descending thoracic aorta: a case report and literature review of surgically treated cases. $J$ Vasc Surg. 1996;24:693-7. 